Wilfried Hommers, Universität Würzburg

\title{
Die Heidelberger Marschak-Interaktionsmethode (H-MIM): Eine kritische Analyse aus familienrechtspsychologischer Sicht.
}

\section{Zusammenfassung}

Der Beitrag fasst zunächst den Hintergrund und die überarbeiteten Inhalte des Manuals der Heidelberger Marschak-Interaktionsmethode (H-MIM) zusammen. Darüber hinaus werden die Durchführung und die Auswertung des Verfahrens dargestellt. Es wird dabei u.a. auf die fehlenden Belege für die Objektivität, Reliabilität und Validität im Manual eingegangen. Weiter wird aufgezeigt, dass bislang kaum empirische Befunde zur Anwendung in der Familienrechtspsychologie existieren. Dennoch kommt der Autor zum Schluss, dass es sich um ein vielversprechendes Verfahren handelt. Unter der Berücksichtigung der Debatte um die Qualität von Gutachten gibt der Autor abschließend Empfehlungen für die Durchführung und Auswertung in der familienrechtspsychologischen Praxis.

Stichworte: Heidelberger Marschak-Interaktionsmethode (H-MIM), psychologische Diagnostik, soziale Interaktion, Familienrechtspsychologie

The Heidelberg Marschak Interaction Method (H-MIM):

A critical analysis from a family law perspective.

\section{Abstract}

The article first summarizes the background and the revised contents of the manual of the Heidelberg Marschak Interaction Method (H-MIM). In addition, the application and evaluation of the procedure is presented. It will point among others to the lack of evidence for objectivity, reliability and validity in the manual. Furthermore, it is shown that there are hardly any empirical reports on application in family law psychology. Nevertheless, the author comes to the conclusion that this is a promising procedure. Taking into account the debate on the quality of expert reports, the author finally gives recommendations for the implementation and evaluation in family law psychological practice.

Keywords: Heidelberg Marschak interaction method (H-MIM), psychological diagnostics, social interaction, family law psychology 


\section{Einleitung und Hintergrund}

Die neu bearbeitete und erweiterte Neuauflage der H-MIM dient wie schon die vergriffene Erstausgabe (Ritterfeld \& Franke, 1994; Franke, 2019) der Anleitung zur Beobachtung der dyadischen Interaktion zwischen einem Kind und seiner erwachsenen Bezugsperson. Die Neuauflage berücksichtigt nun, dass die Interaktionen mit der Bezugsperson vom Alter des Kindes abhängen. Der Bezugsperson werden zwecks Interaktionsbeobachtung Aufgaben für die Gestaltung der Interaktion mit dem Kind gestellt. Diese sind jeweils spezifisch und in unterschiedlicher Anzahl für die vier Altersgruppen inhaltlich verfasst und sind als Einzelkarten in einer DIN-A6-Karteibox separat und nach den Altersbereichen geordnet aufbewahrt. Zuvor waren schon altersbezogene Aufgaben für Kleinkinder, Schulkinder und Jugendliche separat erschienen. Durch diese weiteren Sammlungen wurde die Anwendung der H-MIM schon vor dieser Neuauflage bis in den Schulbereich ausgedehnt (Plattner, 2017).

Die Aufgaben wurden von den Autoren drei Themenbereichen zugeordnet, die mit „Emotionalität“, „Führung“ und „Umgang mit Stress“ überschrieben sind. Während es in der Erstausgabe dafür 18, 22 und 5 Aufgaben (für Kindergartenkinder) gab, bietet die jetzt vorliegende Version unterschiedliche Aufgaben für vier Altersbereiche: Für Kleinkinder oder entwicklungsbehinderte Kinder (im Alter von 4 Monaten bis 3 Jahre) liegen 15, 12 bzw. 6 Aufgaben vor, für Kindergartenkinder (3 bis 7 Jahre alt) 16, 20 bzw. 7 Aufgaben, für Schulkinder (bis zum Alter von 12 Jahren) 12, 9 bzw. 6 Aufgaben und schließlich für Jugendliche (ab dem Alter von 13 Jahren bis ins frühe Erwachsenenalter) 23, 12 bzw. 9 Aufgaben (Aufgabenanzahlen jeweils in der Reihenfolge „Emotionalität“, „Führung“ bzw. „Umgang mit Stress“). Auf den einzelnen DIN-A6-Anweisungskarten ist die Zuordnung zu den Themenbereichen durch die Buchstaben „e“, "“ und „" rückseitig erkennbar. Zusätzlich sind die Aufgaben innerhalb dieser drei Klassen nummeriert.

\section{Das Manual}

Die ersten vier Kapitel des Manuals in Spiralbindung befassen sich mit der Entstehung, den Konstruktionsprinzipien und den gedachten Anwendungen im klinischen Bereich (Diagnose, Indikation und Veränderungsmessung), in der Elternberatung, in Forschung und Ausbildung und schließlich als „Entscheidungshilfe in der familienrechtlichen Begutachtung" (S. 24), worauf das Kapitel 15 noch näher eingeht.

Abgesehen von einführenden Bemerkungen zur Methode der Beobachtung werden die Psychoanalytiker Winnicott, Adler und Dreikurs hier unter dem Stichwort Theoretische Ansätze genannt.

Die beiden Autoren führen in diesen Kapiteln weiter aus, dass sie in der H-MIM fünf Merkmale verwirklicht sehen: (1) Dreiklang aus Kind, Bezugsperson und Zusammenwirken, (2) großes Spektrum der beobachtbaren Aspekte, (3) gezieltes Hervorrufen bestimmter Verhaltensweisen, (4) ressourcenorientierte Auswertung und (5) stressarme Untersuchung. Der Standort der H-MIM sei zwischen standardisierten Tests und 
der ganz freien Beobachtung positioniert. Es gebe weder Skalen noch normative Tabellen, weil sich das soziale Miteinander nicht in Normen fassen lasse. Normen würden vermitteln, das es ein Richtig und ein Falsch, ein Gut oder Schlecht gebe. Das wird mit der Individualität von Aktionen und Reaktionen und in einem Beispiel begründet.

Die drei Aufgabenkategorien werden wie folgt zusammenfassend vor einer detaillierten Darstellung umschrieben:

- (1) Emotionalität mit „beziehungsorientierte Verhaltensweisen als Voraussetzung für Nähe, Bindung und unmittelbarer Einfluss auf das Nervensystem“,

- (2) Führung mit „aufgabenorientierte Verhaltensweisen als Voraussetzung für Lernen, Sicherheit und Entwicklung " und

- (3) Umgang mit Stress wird damit umschrieben, dass dieser Bereich helfe, „von Herausforderungen und Problemen zu lernen und das Nervensystem nicht dauerhaft zu belasten“, wobei das Gelingen „auf den Bereichen der Emotionalität und Führung“ basiere. Aus diesem Dreiklang resultieren Selbstbild und Selbstvertrauen und die Entwicklung des Kindes (Abbildung 4, S. 17).

In den sieben zentralen Kapiteln (Kapitel 5 bis 11 auf S. 28 bis 136) werden die Aufgaben und die Durchführung unter Differenzierung in die vier Altersgruppen detailliert erläutert. Die Aufgaben sind dort auch „Entwicklungspsychologischen Aspekten“ zugeordnet, die ausführlich inhaltlich dargestellt werden und sich zwischen den Altersgruppen in den Inhalten und deren Anzahlen unterscheiden: zwölf (S. 43 f.), zehn (S. 64 ff.), vier (S. 94 f.) oder zehn Aufgaben (S. 113 ff.) in der Folge des Alters der vier Gruppen. Die Zuordnungen der Aufgaben zu den entwicklungspsychologischen Aspekten werden in Tabellen zusammengefasst und durch deren Kennzeichnungen mit den Buchstaben „e“, „f“ und „s“ und folgender Nummer angegeben: Tabelle 2 (S. 44), Tabelle 3 (S. 66), Tabelle 5 (S. 95) und Tabelle 6 (S.116) in der Reihenfolge der vier Altersbereiche. Einige Aufgaben sind jeweils mehreren entwicklungspsychologischen Aspekten zugeordnet.

Das umfangreiche zwölfte Kapitel behandelt die Auswertung (S. 138 bis S. 161). Die ersten fünf Abschnitte (Abschnitte 12.1. bis 12.5.) befassen sich mit allgemeinen Aspekten. Zehn „Zusätzliche Fragen zu Kind und Bezugsperson“ (Abschnitt 12.2., S. 139 ff.) führen in die zu beachtenden Gesichtspunkte und Begriffe bei der Anwendung der H-MIM ein. Eine zugehörige Kurzdokumentation im Anhang (S. 204) enthält statt der in Abschnitt 12.2. dargestellten zehn Fragen zwölf.

Die Abschnitte 12.6. bis 12.11. behandeln konkrete Hilfsmittel und Anleitungen für die summarische Auswertung. Dabei können Tabellen zu einem Überblick verhelfen:

- Die Tabelle 8 (S. 149) beschreibt mit verbalen „Ankern“ unterlegte sechs dreistufige Beurteilungen von Verhaltensweisen des Kindes (Bindung, Impulskontrolle, Wahrnehmung der Umgebung gegenüber, Zielgerichtetes Verhalten, Sprachvermögen und Moralisches Verhalten).

- Die Tabelle 9 (S. 159) benennt 28 Verhaltensweisen aus vier Bereichen, von denen die ersten drei Bereiche den für die H-MIM typischen Aufgabengruppen Emotionalität, Führung und Stress zugeordnet sind und die vierte über alle Aufgaben hinweg

RPsych 5. Jg. 3/2019 
anzuwenden sei. Die Verhaltensweisen werden außerdem als „Bedeutsame Variablen bei der Auswertung der H-MIM“ überschrieben und im Anhang (S. 206) als achtstufige Skalen zum Zweck des Kopierens vorgelegt.

- Die Tabelle 10 (S. 160) stellt die Unterschiede zwischen H-MIM und der US-MIM heraus. Daran schließt auch die Darstellung einer finnischen Auswertung an.

Weiterhin sind elf „Beobachtungsaspekte“ in den Kästen 1 bis 4 (S.151 und 152) zu finden, die im Anhang auch als Kopiervorlage „Leitfaden zur Passung der Interaktion“ zusammengefasst sind. Sieben davon sind auf die Aufgabengruppen zu Emotionalität, Führung und Umgang mit Stress bezogen. Die elf Beobachtungsaspekte umfassen (1) fünf zur Bezugsperson, von denen zwei auf die Aufgabengruppen zur Emotionalität und Führung und weitere drei auf den Interaktionsstil, die Empathie und die Hilfestellungen der Bezugsperson bezogen sind, (2) vier zum Kind, von denen zwei wieder auf die Aufgaben zur Emotionalität und Führung und außerdem zwei weitere auf den Interaktionsstil des Kindes und seinen Umgang mit Stress bezogen sind und (3) schließlich zwei mit insgesamt vier Einzelaspekten zur Interaktion zwischen Bezugsperson und Kind, von denen drei zur Passung der Verhaltensweisen zwischen Kind und Bezugsperson in den drei Aufgabenarten und die andere auf die Entwicklungsangemessenheit des Verhaltens der Bezugsperson bezogen sind. Die kategorial unterlegten Bewertungen enthalten jeweils eine ideale Kategorie und eine oder mehrere als unpassend zu sehende Kategorien, wie in den klein gedruckten Anmerkungen zu den Kästen verdeutlicht wird.

In einem besonderen Abschnitt (12.7., S. 153 ff.) wird die umfänglichere „deskriptive Verlaufsauswertung" beschrieben. Hervorzuheben ist hier das ausführliche Beispiel über die Interaktionen eines vierjährigen Jungen mit seiner Mutter und seinem Vater.

Die Kapitel 13 und 14 befassen sich mit dem die Interaktionsbeobachtungen nachbereitenden Gespräch mit den Bezugspersonen und mit deren Beratung. Das Gespräch mit den Bezugspersonen sei nicht unbedingt in der familienrechtspsychologischen Anwendung der H-MIM vorzunehmen, dem das Kapitel 15 gewidmet ist (vgl. unten).

Im Kapitel 16 wird von Frau Barbara Lleras eine ausführliche Beschreibung von „Theraplay - eine körpernahe Spieltherapie für sozio-emotional und kommunikative gestörte Kinder" gegeben -, an deren Beginn die Interaktionsdiagnostik mit der HMIM und nachfolgendes Gespräch mit der Bezugsperson stehen.

Im Anhang, dem Kapitel 17, findet man altersspezifische Überblicke über die Aufgaben und mehrere Kopiervorlagen. Dabei wird auch darauf hingewiesen, was für die Durchführung der Aufgaben, z. B. durch die von der Untersucherin vorab zu besorgenden Materialien, erforderlich ist. Die DIN-A4-Kopiervorlagen sind für die Planung der Untersuchung, zur Kurzdokumentation der Beobachtungen (jeweils spezifisch für die vier Altersgruppen und einen für alle Altersgruppen zu verwendenden zwölfgliedrigen) vorgesehen. Für die Auswertung der Beobachtungen gibt es zwei Kopiervorlagen, einen elfgliedrigen Leitfaden zur Passung der Interaktion und eine Kopiervorlage für die 28 achtstufigen von „intensiv“ zu „gar nicht“ gerichteten Einschätzungsskalen von „Bedeutsamen Variablen bei der Auswertung der H-MIM“. Schließlich gibt es 
dort eine Kopiervorlage für die zwingend erforderliche Einwilligungserklärung zur Videoaufzeichnung

\section{Durchfübrung}

Empfohlen wird von den Autoren für den diagnostischen Regelfall, je zwei Aufgaben aus den Bereichen „Emotionalität“ und „Führung" sowie eine Aufgabe aus dem Bereich „Umgang mit Stress“ auszuwählen (S.37). Bei schwer einschätzbaren Kindern könne auf je drei Aufgaben aus „Emotionalität“ und „Führung“ erweitert werden. Die einleitende „Eisbrecher-Aufgabe“ soll der Dyade durch ihre Handlungsaktivitäten beidseitige Stressreduktion ermöglichen. Die Aufgabe zu „Umgang mit Stress“ selbst soll die mittlere in der Reihenfolge sein und auf sie soll eine Aufgabe zur „Emotionalität" zum Abklingen des Stress folgen.

Die vorab auf den Fall abgestimmt ausgewählten Karten für die Anweisung der Aufgaben sollen in Briefumschlägen der Bezugsperson in der Reihenfolge der Durchführung sichtbar geordnet übergeben werden. Bei zwei zu untersuchenden Bezugspersonen sei es sinnvoll für diese unterschiedliche Aufgaben zur Vermeidung von Langweile und Verweigerungshaltungen beim Kind auszuwählen. Aber die auf unterstützende Ziele gerichtete, diagnostisch tätige „Therapeutin“ könnte das gegebenenfalls auch dazu nutzen Aufschluss darüber zu erlangen, mit welchen Aufgaben die Bezugspersonen am besten ihre Kompetenzen zeigen können (S. 38). Die meist bekannten Defizite des Kindes wären in der Regel nicht von besonderem Interesse.

Die Bearbeitung der Aufgaben erfolgt an einem Tisch und dient als Grundlage der $\mathrm{zu}$ beobachtenden Interaktionen von Kind und Bezugsperson. Obligatorisch sei die Videoaufnahme der Interaktionen. Zusätzlich sollte zum Zwecke der erleichterten Auswertung, wenn möglich durch geeignete Vorkehrungen (Einwegscheibe oder Übertragung auf einen Monitor in einen Nebenraum), eine das Geschehen begleitende teilnehmende Beobachtung erfolgen. Im Falle des Nichtverfügens über solche Einrichtungen sei es auch akzeptabel, wenn die Untersucherin aus einer Ecke des Raumes unauffällig teilnehmend beobachte.

\section{Zum Anwendungsbereich in familienrechtlichen Begutachtungen}

Der Anwendungsbereich der H-MIM wird aus Sicht der Autoren ursprünglich und maßgeblich in der Diagnostik zur Beratung von Bezugspersonen, für körpernahe Spieltherapie (Kapitel 16) oder für heilpädagogische (Logopädie) Maßnahmen gesehen.

Plattner (2017, S. 23) äußerte sich dahin, dass die H-MIM „für Kinder ab dem Vorschulalter bis etwa zum 13. Lebensjahr im Rahmen einer familienpsychologischen Begutachtung" durchgeführt werden könne. Auch Salzgeber et al. (2018, S. 34) meinten, die schon in der Erstausgabe der H-MIM (Ritterfeld \& Franke, 1994) enthaltenen Aufgaben aus den drei inhaltlichen Schwerpunkten würden sich als „Orientierungshilfen für eine unstrukturierte Beobachtung" eignen. Sie stellten dem aber einen an anderen

RPsych 5. Jg. 3/2019 
Erziehungszielen orientierten Beobachtungsbogen zur kriteriengelenkten Auswertung einer Eltern-Kind-Interaktion bei Seite. Die dort durch eine Auswertungstabelle nach Tausch und Tausch mit 26 sechsstufigen Schätzskalen angegebenen Beurteilungsinhalte sind: Emotionale Wärme (der Mutter/ des Vaters), Lenkung (Mutter/ Vater), Stimulierung des Kindes (Mutter/ Vater), Aktivität (Mutter/ Vater), Harmonische Beziehung (Mutter/ Vater), Bekräftigungsverhalten (Mutter/ Vater) und Spontanität des Kindes (Salzgeber et al., 2018, S. 35-36).

In der vorliegenden 2. überarbeiteten Auflage der H-MIM wird nun die familiengerichtliche Anwendungsmöglichkeit ausdrücklich im „Kapitel 15: Die H-MIM in der familienrechtlichen Begutachtung" auf den Seiten 172 bis 176 behandelt. Die Inhalte des Kapitels 15 wurden gemäß Titelblatt und Danksagung (S. 178) unter Mitarbeit der "Psychologinnen" und Sachverständigen Frau Beatrice Wypych und Frau Dr. Anita Plattner erstellt. Der Text enthält zwei Themenkomplexe: Besonderheiten des Vorgehens in einer Begutachtung sowie ein Praxisbeispiel zu "Scheidung und Sorgerecht" mit einem überlassenen Gutachtenfall seitens der Erstgenannten der beiden unterstützenden „Psychologinnen“.

Die zwischen Vater und dreieinhalbjähriger Tochter erfolgten Interaktionen in fünf Aufgaben werden auf den Seiten 174 und 175 beschrieben. Für die Vater-Tochter-Interaktion wurden folgende fünf Aufgaben aus denen für Kindergartenkinder ausgewählt: „Emotionalität 11: Lassen Sie Ihr Kind auf Ihren Knien reiten“, „Fürsorge 7: Erzählen Sie Ihrem Kind von der Zeit, als es noch ein Baby war“, „Stress 4: Verlassen Sie für eine Minute den Raum und lassen Sie ihr Kind zurück“, „Fürsorge 19: Lassen Sie von Ihrem Kind nachmachen, wie Sie Grimassen schneiden“, „Emotionalität 1: Spielen Sie gemeinsam etwas, was Sie beide kennen“.

Den Interaktionsbeschreibungen folgten positive Wertungen als Anmerkungen und als Gesamtergebnis dann noch, die Beziehung des Kindes zum Vater sei emotional warmherzig, der Vater habe sie gut führen können und habe die jeweilige Befindlichkeit des Kindes sicher erkannt und habe einen guten emotionalen Kontakt zum Kind hergestellt. Dies sei der Mutter weniger gelungen. Über das demnach schlechtere Abschneiden der Mutter in der H-MIM-Anwendung wird aber nichts detailliert berichtet.

Als Besonderheiten der Anwendung im familienrechtspsychologischen Bereich wurden herausgestellt:

- die Auswahl der Aufgaben sollte nach den Erstgesprächen mit den Beteiligten und dem Kennenlernen des Kindes erfolgen;

- wichtig sei gegebenenfalls, die Settings in den jeweiligen Wohnungen der Eltern möglichst ähnlich zu gestalten, bei Umgangsproblemen sei als neutraler Ort die Praxis der/des Sachverständigen zu wählen, in beiden Fällen sei eine Dokumentation durch Videoaufnahme und Beobachtungsnotizen anzustreben;

- bei hochstrittigen Eltern sei die Verwendung der gleichen Aufgaben wegen besserer Vergleichbarkeit zu empfehlen, wofür ein Beispiel auf S. 173 mit E13 und E14 für 
Kindergartenkinder gegeben wird, und bei diesen Eltern sei der Fokus der Aufgaben auf soziale und emotionale Bereiche sinnvoll;

- Aufgaben, die die Kognition ansprechen, sollten nicht für die beiden Dyaden identisch gewählt werden;

- wegen Objektivität und Neutralität gegenüber den (streitenden) Bezugspersonen könne ihnen keine detaillierte Rückmeldung gegeben werden, was in den anderen Anwendungsbereichen gerade dem eigentlichen Ziel der H-MIM-Anwendung entspräche (siehe Kapitel 14 zur Beratung), allenfalls sei Rückmeldung bei Anwendung zur Klärung einer Kindeswohlgefährdung in beschränktem Maße möglich;

- im Einzelgespräch sollte mit der Bezugsperson aber geklärt werden, ob die beobachteten Verhaltensweisen und Interaktionen als typisch für den Alltag mit dem Kind angesehen werden können;

- anerkannt wird ausdrücklich die Ergänzungsbedürftigkeit der Ergebnisse der Anwendung der H-MIM durch andere Befunde aus der Begutachtung, ohne dass dazu weiteren Ausführungen folgen.

\section{Bewertung seitens des Verfassers}

Das einzige und nur spärlich im Kapitel 15 des H-MIM-Manuals ausgeführte Beispiel aus einer Begutachtung einer als Sachverständige bezeichneten „Psychologin“ kann die Auseinandersetzung mit Vorzügen und eventuellen Schwächen einer Anwendung in den rechtlichen Fragestellungen nicht entbehrlich machen.

Das Verfahren für die Beobachtung von Interaktionen zwischen Kind und Bezugsperson H-MIM wurde für die Beratung der Bezugsperson des Kindes entwickelt. Die Übertragung einer Anwendung eines klinischen Verfahrens in die familienrechtspsychologische Begutachtung kann aber sogar dann als maßgeschneidert bezeichnet werden, wenn dabei die Gleichbehandlung von gegebenenfalls zu vergleichenden Bezugspersonen gewährleistet ist. Dazu müssen die ausgewählten Aufgaben aus der H-MIM für die zu vergleichenden Bezugspersonen hinreichend gleichartig sein. Für das Problem der Gleichbehandlung beim Vergleich von strittigen Eltern sollten zumindest angenähert „parallele“ Aufgaben für alle Altersgruppen verfügbar sein. Um hinreichend „parallele“ Aufgaben notfalls selbst zu erzeugen, kann man sich an dem Beispiel auf S. 173 orientieren. Aber es ist unklar, ob man sich unter einer an die H-MIM angelehnten Anwendung eigene Aufgaben ausdenken oder die vorhandenen umformen dürfte, wenn sie sich den drei Bereichen in der vorgesehenen Kombination 2-2-1 oder 3-3-1 zuordnen lassen und der zu begutachtenden Familiensituation gerecht werden.

Folgend wird erörtert, ob bzw. wie weit dem Anspruch zugestimmt wird, dass die H-MIM auch als eine Entscheidungshilfe in der familienrechtlichen Begutachtung dienen könne. Die Grundlage dafür bilden die allgemeine Vorgehensweise der H-MIM, die Angaben in dem diesem Thema gewidmeten Kapitel 15 der H-MIM und die allgemein für die H-MIM vorgesehene Auswertung.

RPsych 5. Jg. 3/2019 
Diese Bewertung erfolgt vor den allgemeinen methodischen Anforderungen an ein Verfahren (Standardisierung der Durchführung, Kriteriengeleitete Auswertung, Objektivität der Auswertung, Validität der Schlussfolgerungen) und den besonderen familienrechtspsychologischen Kriterien (Gleichbehandlung, Nachvollziehbarkeit, Bezug zum gerichtlichen Auftrag wie Aufenthalt, Umgang oder Gefährdung des Kindeswohls), denen die H-MIM hinreichend genügen sollte.

\section{1 $\mathrm{Zu}$ den Aufgaben}

Zunächst ist festzustellen, dass die H-MIM eine reichhaltige Aufgabenmenge zur Einleitung von Interaktionen bereitstellt. Die drei unterschiedlichen Interaktionsbereiche betreffen Emotionalität, Führung und Umgang mit Stress. Damit erscheint der Grundstock für eine angenähert repräsentative, aber dennoch spezifische Erfassung der Beziehungen zwischen dem Kind und seinen Bezugspersonen gelegt. Auf einem Itemkonstruktionsvorgang wie bei der Erstellung von Tests oder Fragebögen beruhte die Entstehung der in H-MIM enthaltenen Aufgabenmengen für die vier Altersbereiche aber weder auf theoretischer noch auf empirischer Basis. Ob das als Mangel gelten muss, bleibt dahin gestellt.

Darüber hinaus bleibt zu fragen, ob die tatsächlich eingesetzte Menge von fünf oder sieben Aufgaben erschöpfend für die konkrete familiengerichtliche Fragestellung sein kann, und ob die entnommenen fünf oder sieben Aufgaben tatsächlich hinreichend für eine sachgerechte Beurteilung von Bezugspersonen sind, die sich in einem familiengerichtlichen Verfahren befinden. Möglicherweise eignen sich die zur Verfügung stehenden Aufgaben auch nicht alle für familiengerichtliche Anwendungen. Es bleibt schließlich nicht ausgeschlossen, dass es weitere für eine familienrechtspsychologische Begutachtung geeignete Aufgaben zur Auslösung von Interaktionen zwischen Kind und Bezugsperson gibt. Hier muss sich der Sachverständige auf seine Urteilskompetenz verlassen.

Das Kind im Beispiel „Laura-Vater“ entstammt dem Altersbereich Kindergartenalter. Wie sich die Anwendung der H-MIM auf die anderen Altersgruppen ausdehnen ließe, wurde nicht beispielhaft dargelegt. Auch wären Beispiele oder Anleitungen zu anderen gerichtlichen Fragestellungen als dem Streit zwischen Eltern um die Sorge oder den Aufenthalt des Kindes, z. B. zur Kindeswohlgefährdung zu begrüßen.

\subsection{Zur Erfüllung methodischer Anforderungen}

Bei der Interaktionsbeobachtung sei nach Salzgeber et al. (2018, S. 34) wichtig, „dass die Beobachtung systematisch und kriteriengeleitet erfolgt". Dass die H-MIM dazu das Potential hat, kann der H-MIM zuerkannt werden. Unter Bezug auf die Systematik der Beobachtung wurde mit der Standardisierung der Durchführung und der Anforderung Videoaufnahme ein wesentlicher Aspekt erreicht. Das zeigt sich auch in der 
fünf- oder siebengliedrigen Auswahlkombination und der Abfolge 2-1-2 bzw. 3-1-3 für die Aufgaben aus den drei Gebieten.

Die Auswertung erfolgt abgesehen von der „Deskriptiven Verlaufsauswertung“ (12.7., S. 153-158) offensichtlich auch durch Kriterien angeleitet. Das ist in den 28 achtstufig zwischen den Polen „gar nicht“ und „intensiv“ gerichteten „Bedeutsamen Variablen bei der Auswertung der H-MIM“ und dem elfgliedrigen „Leitfaden zur Passung der Interaktion“ zu erkennen. Weiterhin sind die zwölf „Zusätzlichen Fragen zu Kind und Bezugsperson" in dieser Hinsicht als Förderungen der von Kriterien geleiteten Auswertung anzuführen.

Man kann davon ausgehen, dass zu einer Anwendung der H-MIM die Auswertung nach den im Kapitel 12 beschriebenen Elementen gehört. Es stellt sich aber wieder die Frage, wodurch diese Zusammenstellung von Kriterien begründet ist. Ist es zwingend erforderlich, dass die Verhaltensweisen aufgrund der nach dem Ablaufschema der HMIM vorgegebenen Aufgaben mit den drei genannten Gruppen von Kriterien ausgewertet werden? Könnte man unterstellen, dass sich die Beobachtungen mit H-MIMAufgaben auch mit den Tausch\&Tausch-Skalen von Salzgeber et al. (2018, S. 35) auswerten ließen? Die Bereiche Emotionalität und Führung in der H-MIM erscheinen ähnlich zu den beiden Dimensionen des Erzieherverhaltens bei Tausch \& Tausch (1979), die Wertschätzung und die Lenkung. Auch Tausch \& Tausch (1979) befassten sich mit dem Aspekt Stress, indem sie die Verwirklichung optimaler Ausmaße von Wertschätzung und Lenkung in erzieherischen Konfliktsituationen bearbeiteten. Insofern erscheint die Auswertung mit den Tausch\&Tausch-Skalen in Salzgeber et al. (2018) nicht unpassend. Ein empirischer Vergleich dazu erscheint als eine interessante Fragestellung zur konkurrenten Validität.

Unter Bezug auf Salewski \& Stürmer $(2015$, S. 6) lässt sich die methodische Bewertung erweitern. Demnach sei bei Verhaltensbeobachtungen die Systematisierung durch Verwendung eines Beobachtungssystems oder Kodierschemas von zentraler Bedeutung, da mit zunehmender Systematisierung deren Objektivität, Reliabilität und Validität steige. Sie kodierten in ihrer gutachtenkritischen Untersuchung, ob Angaben darüber erfolgten, welche Aspekte des Verhaltens zu welchem Zweck beobachtet wurden und ob aus der Dokumentation eine Zielsetzung hervorging. Sie berichteten dann, dass in 91 von 116 Gutachten diesem Anspruch nicht genügt worden sei. Diesem höheren Anspruch wird das Manual der H-MIM weniger eindeutig gerecht.

Unter Bezug auf Salewski \& Stürmer (2015) gilt die obige positive Bewertung daher nur beschränkt auf die bloße Existenz von Systematisierungen und Kodierschemata. Allerdings bleiben im Manual Objektivität, Reliabilität und Validität der H-MIM-Auswertung völlig ungeklärt. Deutlich wird, dass Hinweise auf die Gütekriterien, der sich jede Methode stellen muss, weder im Manual beschrieben werden, noch durch zitierte Ergebnisse empirischer Studien behauptet werden. Insbesondere die Urteiler-Übereinstimmung (Inter-Rater-Reliabilität) wäre gerade wegen der für Urteilereinflüsse oder -fehler prädestinierten Komplexität des Verfahrens in familiengerichtlichen Verfahren von Interesse. Eine stichhaltige Begründung wurde auch nicht gegeben, sie erschien wohl selbstverständlich. Denn selbst, wenn man meinen würde, dass Interaktionen im-

RPsych 5. Jg. 3/2019 
mer höchst spezifisch für Aufgabe, Kind und Bezugsperson sind, wäre doch nicht einzusehen, warum man nicht die Gleichheit der Ergebnisse zweier Urteiler über die gleiche Videoaufnahme einer Interaktion bestimmen könnte.

Dass das erforderlich und lösbar ist, hatte die Erstautorin der Erstversion belegt. Ritterfeld (1997, S. 82 ff.) berichtete über die „Zuverlässigkeit“ der damaligen Version der H-MIM auf der Basis einer Diplomarbeit von Hölzel aus dem Jahr 1993. Sechs Auswerter kodierten sechs Interaktionssequenzen aus Videobeispielen unabhängig voneinander. Das in der Entstehung schwer nachzuvollziehende Ergebnis (Tabelle 1, S. 85) zeigte schlechtere Inter-Rater-Reliabilitäten (d.h. Objektivitätsmaße) gerade für die „Passung“ an, wie auch von Ritterfeld hervorgehoben. Während für Emotionalität und Führung die Inter-Rater-Reliabilitäten deutlich über 0,60 lagen $(0,72$ und 0,72), betrugen sie bei der Passung deutlich weniger als 0,60 (0,56 im Gesamturteil sogar nur 0,50). Interessant ist auch, dass Ritterfeld (1997, S. 84) nicht von Interaktionsdiagnostik, sondern nur von Interaktionsbeschreibung sprechen möchte. Gleichwohl stelle diese wegen ihrer evaluativen Komponente „den unmittelbaren Ansatzpunkt für die therapeutische Intervention als Annäherung an den Idealtyp“ bereit. Diesem Beispiel wäre unbedingt mit einer umfassenden Untersuchung zu der Objektivität der Auswertungsvariablen in der Neuauflage nachzustreben.

Zwar schützen selbst die anscheinend höheren Inter-Rater-Reliabilitäten für Emotionalität und Führung nicht vor Fehlbeurteilungen eines Beobachters in einem konkreten Fall, aber geringe Inter-Rater-Reliabilitäten wie zu der Passung der Interaktion würden nahe legen, dass die H-MIM zumindest dort nicht zu verlässlichen Ergebnissen führen würde, weil der unsystematische Einfluss des Beobachters auf das Ergebnis $\mathrm{zu}$ groß ist. Vermutlich meinen die Autoren unzulässiger Weise, wie mit der Ablehnung von Normen (Abschnitt 2.4.4.) geschehen, auch die Bestimmung von Inter-Rater-Reliabilitäten, die sie aber nicht einmal gedanklich in Betracht gezogenen haben, stichhaltig ablehnen zu können. Solange in der Bestimmung der Inter-Rater-Reliabilität aber nicht angemessen nachgebessert worden ist, kann die H-MIM daher nicht den Status eines empirisch-wissenschaftlich ausgewiesenen Verfahrens beanspruchen. Der Anwender sieht sich damit bei Verwendung der H-MIM in einem familienrechtspsychologischen Gutachten gar nicht ausreichend durch Forschungsergebnisse gestützt, quasi in einem methodenkritischen Leerraum.

Ähnlich verhält es sich bei den Reliabilitäten von insgesamt vier möglichen Summenwerten aus den 28 wertend gerichteten achtstufigen „Bedeutsamen Variablen“. Diese wären zwar ebenfalls durch empirische Untersuchungen noch zu klären und könnten über den Nachweis von ausreichenden Ausprägungen von Cronbachs Alpha die systematische Auswertung verbessern. Aber hier wäre erforderlich, dass eine möglichst an familienrechtspsychologischen Fallmustern orientierte Standardmenge von Aufgaben mit einer hinreichend umfangreichen Menge von Interaktionspaarungen an einer Norm-Stichprobe und einer klinischen Stichprobe untersucht würde, damit ein grundlegender Eindruck über die Reliabilität von individuellen Summenwerten aus den „Bedeutsamen Variablen“ besteht. Das mag wegen der individuellen Ausprägung 
von familiengerichtlich anfallenden Problemstellungen nicht immer einfach sein. Aber auch hier fehlt es eindeutig an diagnostischer Grundlagenforschung.

Allerdings wird in der H-MIM-Auswertung anscheinend nicht angestrebt, Summenwerte aus den 28 „Bedeutsamen Variablen“ zu bilden. Unklar bleibt dann aber, wie die vielen Beurteilungsvariablen aus den 28 „Bedeutsamen Variablen“, aus den Aspekten des Leitfadens zur Passung und aus den zwölf zusätzlichen Fragen von einem Anwender zu einem Gesamturteil integriert werden können. So wie es jetzt erscheint, bleibt das Gesamturteil oder die Schlussfolgerung völlig der durch nichts angeleiteten klinischen Beurteilung aus der „Deskriptive Verlaufsauswertung“ (siehe 12.7. im Manual) überlassen. Demnach bliebe aber die Frage, welchen Stellenwert haben die vielen Beurteilungsvariablen (28 Skalen, Leitfaden zur Passung, 12 zusätzlichen Fragen) in der Auswertung. Könnte die Auswertung hinsichtlich der Integration aller Einzelskalen weiter systematisiert werden oder ist sie nur Beiwerk?

Auch die Stabilität durch Bestimmung von Retest-Korrelationen könnte besonders im Falle von Aufträgen zur Kindeswohlgefährdung die Schlussfolgerungen aus defizitärem Interaktionsverhalten der Bezugsperson das Gewicht aus den Folgerungen aus der Anwendung der H-MIM stützen. Die Übertragung der relativ hohen Retest-Korrelationen aus der im Internet zugänglichen Längsschnittstudie zur therapeutisch bedingten Veränderungsmessung von Franke und Wettig (2005) erscheint zwar viel versprechend ( 9 von 10 signifikant zwischen $r=0,47$ und $r=0,70$ ). Aber abgesehen von einigen Unklarheiten im Untersuchungsbericht von Franke und Wettig (2005) ist wegen der dortigen andersartigen Skalen, wegen der zahlreichen methodischen Unterschiede und wegen der unterschiedlichen Auswertungsvariablen zwischen der 2. Version der H-MIM und dem von Franke und Wettig (2005) erfolgtem Vorgehen die Übertragung dieser Ergebnisse zur Re-Test-Korrelation nicht möglich.

Untersuchungen zur Validität des Verfahrens sind weiterhin außerhalb therapeutisch-indikativen Anwendungen der H-MIM erforderlich. Hinsichtlich der konkurrenten Validität wäre unter familienrechtspsychologischer Perspektive von Interesse, die 28 achtstufigen H-MIM-Skalen mit den 26 sechsstufigen Skalen der Auswertungstabelle bei Salzgeber et al. (2018, S. 35-36) zu vergleichen. Von Salzgeber et al. (a.a.O.) werden für die Skalen der auf Tausch \& Tausch zurückgehenden Auswertungstabelle auch keine Angaben zur Inter-Rater-Reliabilität oder zur Integration usw. genannt. Diese würden sich aber im Falle hoher Korrelationen zwischen beiden Auswertungsverfahren unterstellen lassen. Solche empirischen Studien würden auch erste empirische Normwerte wie Mittelwerte und Standardabweichungen ergeben.

Zur Normierung vertreten die Autoren der H-MIM im Manual zwar die Ansicht, dass es in einer Interaktionsdiagnostik, anders als in psychometrischen Tests weder Skalen noch normative Tabellen geben könne, weil sich das individuelle soziale Miteinander nicht in Normen fassen lasse. „Normen würden hier vermitteln, dass es ein Richtig und ein Falsch, ein Gut oder Schlecht gibt" (S. 14). Die Autoren vertreten demnach einen wertenden Normen-Begriff. Darin liegt aber ein erhebliches Missverständnis der deskriptiven Normen für Untersuchungsverfahren verborgen, um die es aus empirisch wissenschaftlicher Sicht geht. Weiterhin gibt es in der H-MIM ange-

RPsych 5. Jg. 3/2019 
sichts der 28 achtstufigen Bedeutsamen Variablen doch Skalen und die elf „Beobachtungsaspekte“ in den Kästen 1 bis 4 (im Anhang als „Leitfaden zur Passung der Interaktion") sind eindeutig wertende Kriterien. Wer mehrstufige Skalen einführt, muss auch die Variabilität der Ergebnisse in Normstichproben kennen, um angemessene Schlussfolgerungen über den Vergleich zwischen Eltern oder über das Unterschreiten der Schwelle zur Kindeswohlgefährdung ziehen zu können.

Daher vertritt der Verfasser die allgemein geteilte Ansicht, dass sich sowohl beim Elternvergleich, als auch bei der Einschätzung der Kindeswohlgefährdung erforderlich und nützlich erweist, über Mittelwerte, Standardabweichungen und Reliabilitäten bzw. Interne Konsistenzen wie bei Testverfahren verfügen zu können.

\section{Fazit}

Ein reichhaltiges und gründlich überarbeitetes Material zur strukturierten Durchführung von Interaktionen zwischen Kindern in vier Altersbereichen und Bezugspersonen wird durch die überarbeitete Neuauflage der H-MIM vorgelegt. Auch in den Details des Aufbaus und der damit verbundenen Auswertung ist ein umfassender entwicklungspsychologischer Ansatz gelungen, der sich als Grundlage einer Elternberatung oder als Ansatz für eine heilpädagogische Maßnahme bei einem weiten Altersbereich von Kindern und Jugendlichen verwenden lässt. Das Manual enthält leider keinerlei Bezug auf empirische Forschungsergebnisse zu den methodologischen Kriterien wie Objektivität, Reliabilität und Validität, die explizit die methodischen Qualität des Verfahrens in den angestrebten Anwendungsbereichen belegen könnte.

Die Anwendung im familienrechtspsychologischen Rahmen erscheint zumindest viel versprechend, weil sie auf ein systematisiertes Verfahren zurückgreifen kann und eine von Kriterien geleitete Auswertung anbietet. Die Anwendung im Rahmen familiengerichtlicher Begutachtungen bedarf aber noch erheblicher Bemühungen jedes Anwenders, um sich aus dem vorliegenden Material fallspezifisch eine zumindest face-valide Vorgehensweise zusammenzustellen. Er kann sich nicht auf empirische Forschungsergebnisse stützen, mit denen er argumentativ methodenkritischen Einwänden begegnen kann. $\mathrm{Zu}$ fordern ist daher einerseits ein ausführlicherer, auf die Altersbereiche und die familiengerichtlichen Fragestellungen bezogener Leitfaden zur Anwendung der H-MIM in der familienrechtspsychologischen Begutachtung, und andererseits sind empirische Untersuchungen zu den einschlägigen Gütekriterien Objektivität, Reliabilität und Validität erforderlich, um grundlegende methodische Anforderungen $\mathrm{zu}$ überprüfen.

Nicht ausgeschlossen erscheint aber, dass die Anwendung von Elementen der $\mathrm{H}$ MIM unter Beachtung der vielen im Manual genannten Anforderungen, insbesondere Videoaufnahme und wiederholte Auswertung u.U. ergänzt durch Dritte, zu gerichtlich übernommenen Empfehlungen führen kann. Das ist umso wahrscheinlicher je mehr sie (1) aufgrund sorgfältiger Vorbereitung seitens der/des Sachverständigen beruht, (2) zwecks Nachvollziehbarkeit in einen ausführlichen Bericht darüber mündet und (3) im 
Verbund mit anderen Befunden gemäß der multimodalen Diagnosestrategie gestützt oder ergänzt wird. Unberechtigt erscheint, das Verfahren mit den vielen projektiven oder semi-projektiven Verfahren, die mit der Zuordnung Explorationshilfen im methodischen Stellenwert geführt werden, gleich zu setzen. Die H-MIM hat zweifellos das Potential, auch zu einer empirisch hinreichend unterlegten, methodenkritisch positiven Einschätzung zu gelangen. Was zur Verbesserung in methodischer Hinsicht und in Hinsicht auf die familiengerichtliche Anwendung wünschenswert oder notwendig erscheint, ist zuvor dargelegt worden.

\section{Literatur}

Franke, U. (2019). Information zu H-MIM. Über Diagnostik des sozialen Interaktionsverhaltens mit der Heidelberger Marschak Interaktions-Methode (H-MIM). https:/ /www.theraplay-institut.de/interaktionsdiagnostik-h-mim (Zugriff am 01.07.2019).

Franke, U. \& Wettig, H.H.G, (2005). H-MIM. Heidelberger Marschak InteraktionsMethode zur Diagnostik der Mutter-Kind Interaktion. Validität - Effektgröße - Sensitivität - Reliabilität. Forschungsergebnisse einer Längsschnittstudie 1998-2005. Leonberg: Theraplay Institut. https://slideplayer.org/slide/212747/ bzw. für Folien 40 bis 42 https://..../5238511/ (Zugriff am 01.07.2019).

Plattner, A. (2017) Erziehungsfähigkeit aus psychologischer Sicht. In A. Plattner (Hrsg.). Erziehungsfähigkeit psychisch kranker Eltern richtig einschätzen und fördern. München: Reinhardt.

Ritterfeld, U. (1997). Beschreibung als Diagnose?! Zur Analyse von Eltern-Kind-Interaktionen. Journal für Psychologie, 5, 77-85. https://nbn-resolving.org/urn:nbn:de:01 68-ssoar-29184 (Zugriff am 30.07.2019).

Ritterfeld, U. \& Franke, U. (1994). Die Heidelberger Marschak-Interaktionsmethode (H-MIM). Zur diagnostischen Beurteilung der dyadischen Interaktion mit Vorschulkindern. Mit einem Anhang der Interaktionsaufgaben im Überblick, Materialien und Formblättern. Stuttgart: Fischer.

Salewski, C. \& Stürmer, S. (2015). Qualität familienrechtspsychologischer Gutachten, Eine aktuelle empirische Studie. Zeitschrift für Kindschaftsrecht und Jugendhilfe, 11, 49.

Salzgeber, J., Bretz, E. \& Bublath, K. (2018). Arbeitsbuch familienpsychologische Gutachten. Arbeitshilfen für ein sachverständiges Vorgeben bei der familienrechtspsychologischen Begutachtung. München: Beck.

Tausch, R. \& Tausch, A.-M. (1979). Erziehungspsychologie (5. gänzlich neu gestaltete Aufl.). Göttingen: Hogrefe. 
Kontakt:

Prof. Dr. Wilfried Hommers

Univ.-Prof. i.R. am Institut für Psychologie

Julius-Maximilians-Universität Würzburg

Email: hommers@psychologie.uni-wuerzburg.de

Postanschrift:

Alter Hettstadter Weg 12

97249 Eisingen 